\title{
On the Calculation of the Inverse of the Error Function*
}

\author{
By Anthony J. Strecok
}

\begin{abstract}
Formulas are given for computing the inverse of the error function to at least 18 significant decimal digits for all possible arguments up to $1-10^{-300}$ in magnitude.

A formula which yields erf $(x)$ to at least 22 decimal places for $|x| \leqq 5 \pi / 2$ is also developed
\end{abstract}

1. Introduction. In statistical work, many types of probability integrals or sums are approximated by functions which involve the normal probability integral or its inverse. Examples where the inverse is used in the asymptotic expansions of $\chi^{2}$ distributions can be found in the first four references which are given at the end of this report. J. R. Philip [5] notes that the solution of a one-dimensional concentration-dependent diffusion equation can be obtained with the aid of the inverse error function, and also suggests some formulas which are useful for computation.

Formulas for the direct computation of the inverse error function have also been discussed by L. Carlitz [6]. Moreover, a computer program which obtains the inverse has recently been designed at the University of Chicago [7].

Throughout the remainder of this paper, we will use the notations

$$
x=\operatorname{erf}(y)=\frac{2}{\sqrt{ } \pi} \int_{0}^{y} e^{-t^{2}} d t \text { and } y=\operatorname{inverf}(x) .
$$

Since some formulas for $y$ are obtained from numerical values of erf $(y)$, it is necessary to consider the calculation of erf $(y)$ also.

2. Formulas for $\operatorname{erf}(y)$. In the well-known Eq. [8]

$$
\sum_{m=-\infty}^{\infty} \exp \left(-K(m+T)^{2}\right)=(\pi / K)^{1 / 2} \sum_{n=-\infty}^{\infty} \exp \left(-K T^{2}+(K T+i n \pi)^{2} / K\right),
$$

we take $K=25 \pi^{2}$ and $T \leqq \frac{1}{2}$ and obtain

$$
e^{-(5 \pi T)^{2}}+\epsilon(T)=\left[1+2 \sum_{n=1}^{37} e^{-(n / 5)^{2}} \cos 2 n \pi T\right] /(5 \sqrt{ } \pi)
$$

where $|\epsilon(T)|<10^{-25}$. If we take $5 \pi T=z$ and integrate with respect to $z$ from 0 to $y$, we see that

$$
\operatorname{erf}(y) \approx \frac{2}{\pi}\left[y / 5+\sum_{n=1}^{37} n^{-1} e^{-(n / 5)^{2}} \sin (2 n y / 5)\right] \text { for }|y| \leqq \frac{5 \pi}{2} .
$$

In order to circumvent the computation of the 37 values of $\sin (2 n y / 5)$, we transform (1) essentially into a polynomial in $\alpha=2 C^{2}-1$, where $C=\cos (2 y / 5)$.

Received September 26, 1966.

* Work performed under the auspices of the U.S. Atomic Energy Commission. 
From trigonometric identities, we have

$$
\sin (2 y(2 n-1) / 5)=S \cdot P_{2 n-1} \text { and } \sin (2 y(2 n / 5))=2 C S \cdot P_{2 n}
$$

where

$$
S=\sin (2 y / 5), \quad P_{m+1}=\left[1+\left(1+(-1)^{m}\right)\left(\frac{1}{2}+\alpha\right)\right] P_{m}-P_{m-1} \quad(m \geqq 2)
$$

with $P_{1}=1=P_{2}$. When we substitute the appropriate $S \cdot P_{2 n-1}$ and $2 C S \cdot P_{2 n}$ expressions into (1) and simplify the result, we obtain

$$
\operatorname{erf}(y) \approx 2 y /(5 \pi)+S \sum_{n=1}^{19}\left(A_{1 n}+2 C \cdot A_{2 n}\right) \alpha^{n-1} .
$$

The coefficients $A_{1 n}$ and $A_{2 n}$ are given in Table 1. These coefficients, as well as all the others given in this report, were computed on the CDC 3600 computer at Argonne National Laboratory.

Formula (2) was checked by comparing numerical values of erf (y) with the results of the series expansion

$$
\operatorname{erf}(y) \approx \frac{2}{\sqrt{ } \pi} y \sum_{n=0}^{25} \frac{\left(-y^{2}\right)^{n}}{n !(2 n+1)}
$$

for $y=10^{-3}\left(10^{-3}\right) 10^{-1}$. The maximum difference between corresponding values was never found to exceed $10^{-23}$ in magnitude.

For $y>2$, we used the continued fraction [9]

$$
\int_{y}^{\infty} e^{-t^{2}} d t=\frac{e^{-y^{2}}}{2 y+} \frac{1}{y+} \frac{2}{2 y+} \frac{3}{y+} \frac{4}{2 y+} \cdots
$$

to obtain

$$
\operatorname{erf}(y)=1-\frac{2}{\sqrt{ } \pi} \int_{y}^{\infty} e^{-t^{2}} d t
$$

The results of (2) and (4) were compared for $y=2(.01) 7.85$, and again no differences between corresponding results were found to exceed $10^{-23}$ in magnitude.

3. The Calculation of $\operatorname{inverf}(x)$ for Small $x$. If primes indicate differentiation with respect to $x$, then from $x=\operatorname{erf}(y)$, we have $1=(2 / \sqrt{ } \pi) e^{-y^{2}} y^{\prime}$, or

$$
y^{\prime}=\frac{\sqrt{ } \pi}{2} e^{y^{2}}
$$

Then

$$
y^{\prime \prime}=2 y y^{\prime} y^{\prime} .
$$

Carlitz [6] has developed a series expansion from a differential equation similar to (6). However, we will proceed in a different manner.

Equation (6) can be written as $y^{\prime \prime}\left(y^{\prime}\right)^{-2}=2 y$ and integrated to produce $-1 / y^{\prime}=2 \int y d x+C$. From (5), it is evident that $y^{\prime}=\sqrt{ } \pi / 2$ when $y=0=x$. 


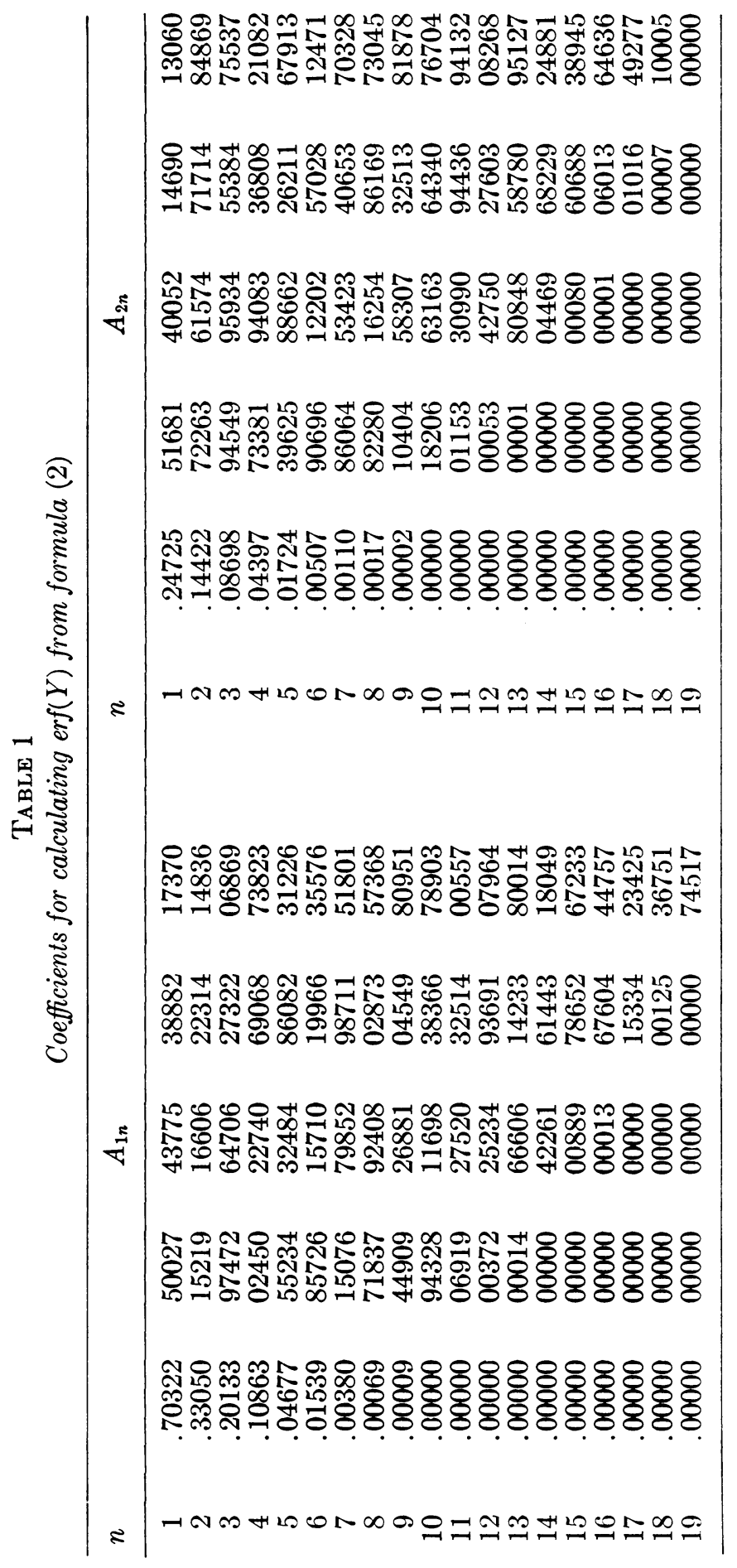


Consequently,

$$
-1 / y^{\prime}(x)=2 \int_{0}^{x} y(t) d t-2 / \sqrt{ } \pi .
$$

Equation (7) can be used for analogue machine computation, since all values at $x=0$ are known.

It may also be noted that if Eqs. (5) and (7) are combined, then

$$
\int_{0}^{x} y(t) d t=\left(1-e^{-y^{2}(x)}\right) / \sqrt{ } \pi .
$$

A similar result which involves inverf $(1-x)$ was obtained by Philip [5].

If we now assume that

$$
\operatorname{inverf}(x)=\sum_{n=1}^{\infty} C_{n} x^{2 n-1}
$$

for small $x$, then from (7)

$$
1+\left(\sum_{m=1}^{\infty}(2 m-1) C_{m} x^{2 m-2}\right)\left(\sum_{n=1}^{\infty} n^{-1} C_{n} x^{2 n}-2 / \sqrt{ } \pi\right)=0 .
$$

The $C_{n}$ values can be determined by multiplying the series of (9) and equating the coefficient of each power of $x^{2}$ to zero.

The first 200 values of $C_{n}$ were computed and are given in Table 2 . No attempt was made to determine the accuracy of these coefficients directly. Instead, Eq. (8) was used in the calculation of

$$
\left.\epsilon_{1}=\mid x^{-1} \text { erf (inverf }(x)\right)-1 \mid
$$

and

$$
\epsilon_{2}=\mid y^{-1} \text { inverf }(\operatorname{erf}(y))-1 \mid
$$

for $x=.001(.001) .875$. In this range, the test calculations have not found any $\epsilon_{1}$ or $\epsilon_{2}$ as large as $10^{-22}$.

Since the operations which produced Eq. (8) are also valid for complex values $x=z$, it should be possible to obtain good results from (8) whenever the inverse is unique. In this way, it should be feasible to obtain the inverse of Dawson's integral $\int_{0}^{y} e^{t^{2}} d t$ or other special functions for small arguments.

The first 200 terms of (8) were telescoped [10] by W. J. Cody, Jr. of Argonne for the range $|x| \leqq .8$. The result, equivalent in accuracy to (8), is expressed in the form

$$
\operatorname{inverf}(x)=x\left\{\xi_{0}+\sum_{n=1}^{38} \xi_{n} T_{n}\left(\frac{x^{2}}{.32}-1\right)\right\},
$$

where $T_{n}(\lambda)$ is the Chebyshev polynomial of degree $n$ in $\lambda$ and the $\xi_{n}$ are the coefficients in Table 3.

4. Asymptotic Forms. Philip [5] suggests using a continued logarithm to obtain inverf $(x)$ for large values of $x$. However, this asymptotic expansion appears to be accurate only for values of $x$ which are very close to unity. 


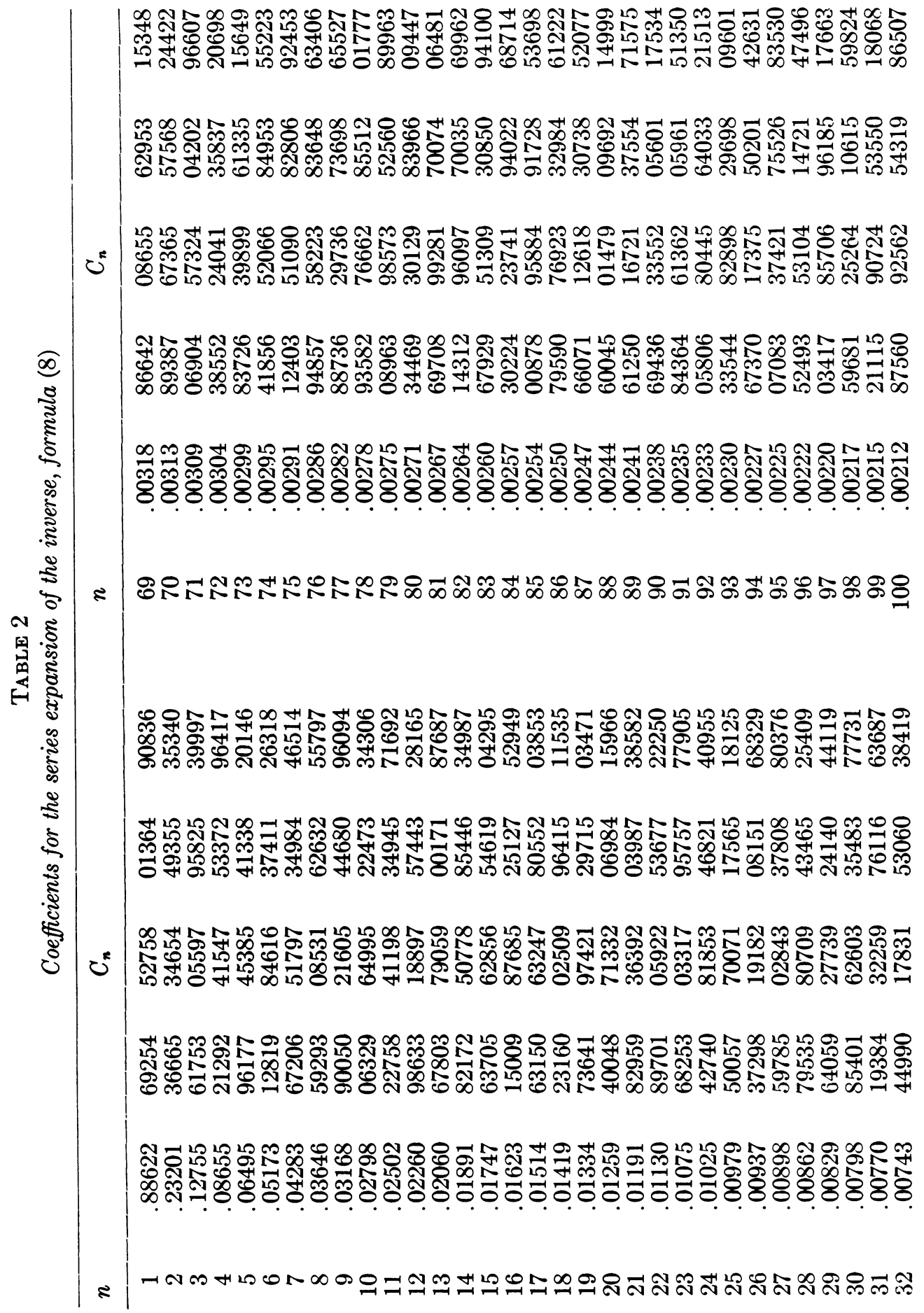


역 운

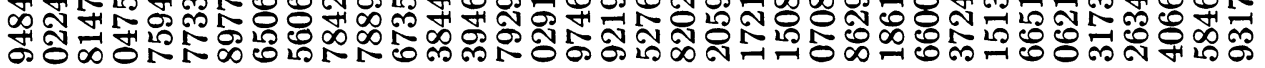

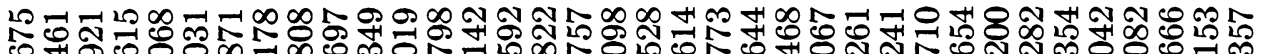



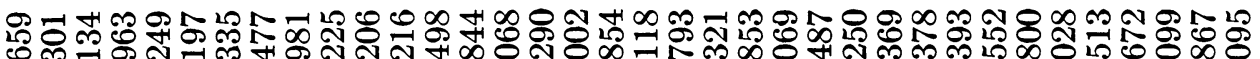

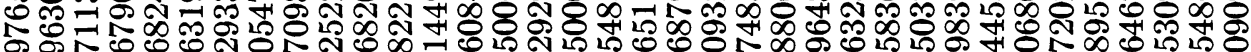

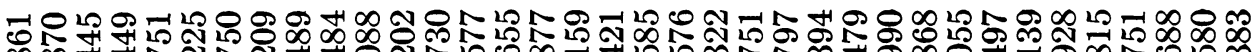
$\infty \infty$ F 品

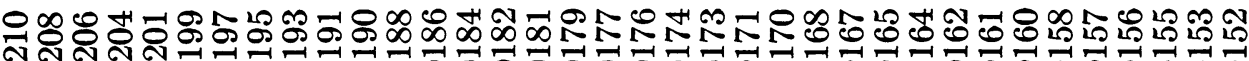

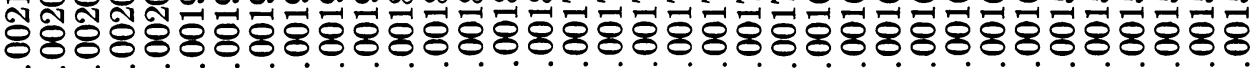

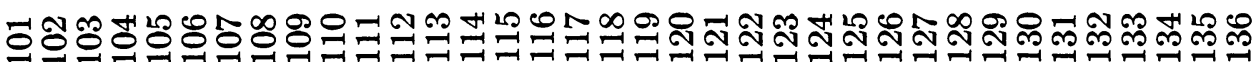

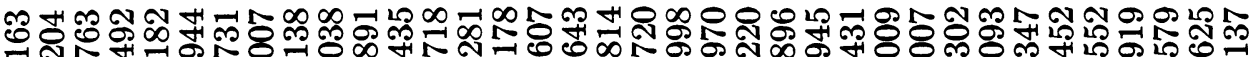

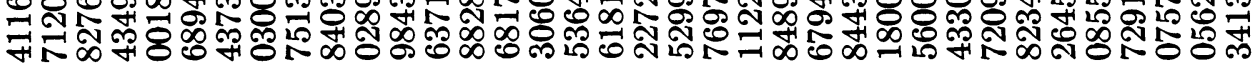

茾占



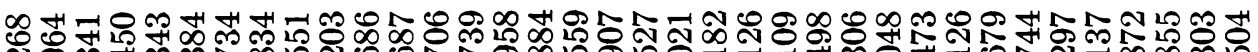
그음 北施

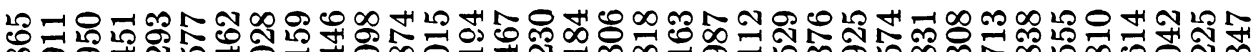

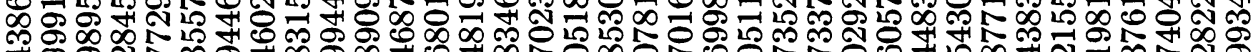

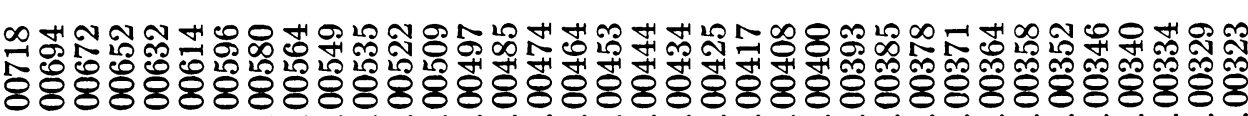

๓ొ 


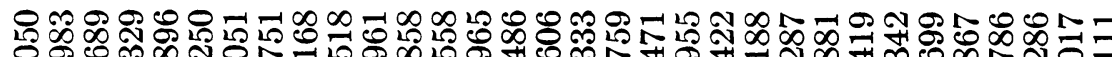


前

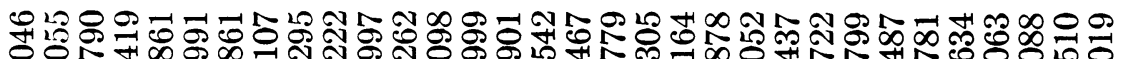

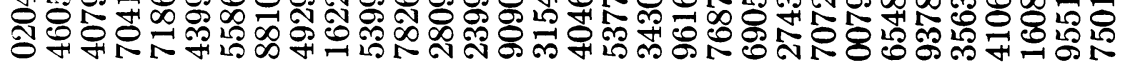

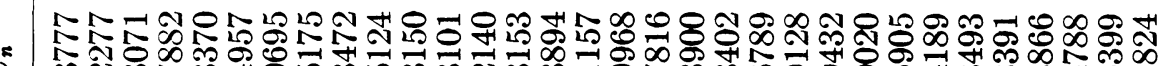
今.

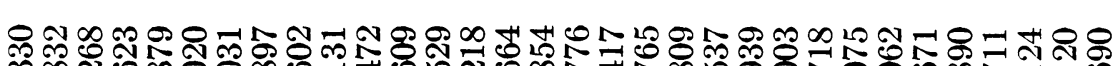



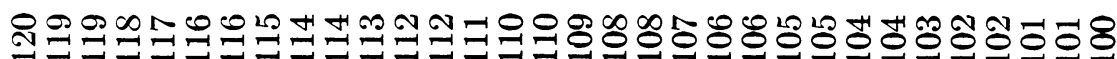



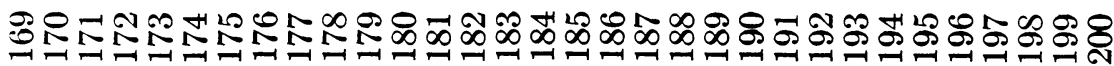

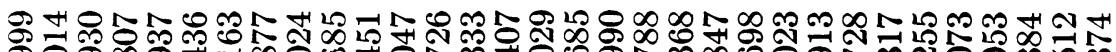

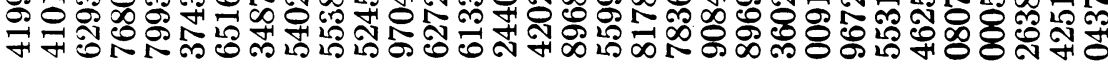

웅



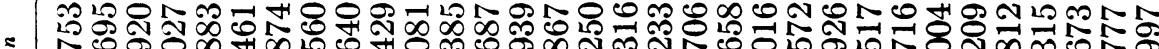

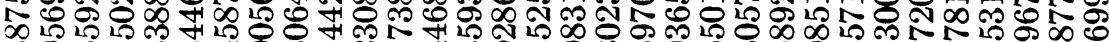
$\infty$ 织

숙순

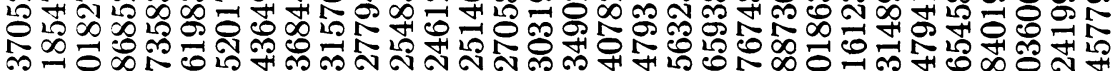

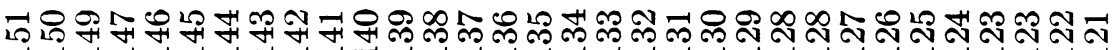

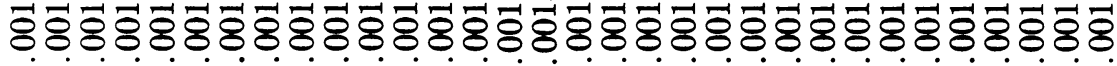

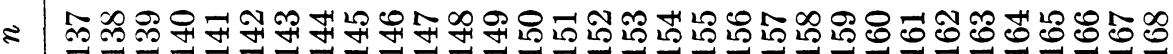


THE INVERSE OF THE ERROR FUNCTION

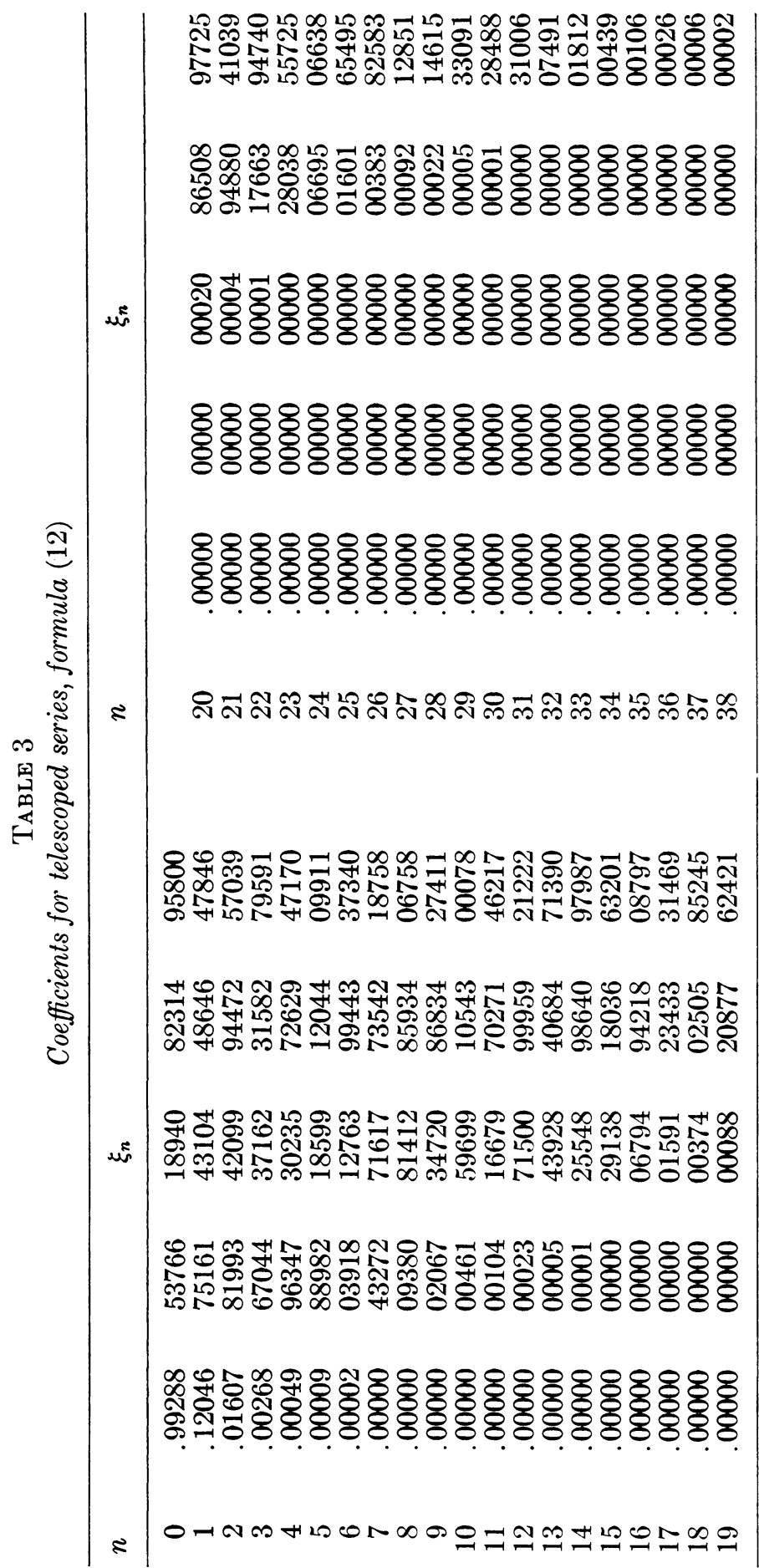


C. Hastings [11] essentially approximates the inverse by using rational functions of $\left(-\ln t^{2}\right)^{1 / 2}$ where $t=1 /(2 \pi)^{1 / 2} \int_{x}^{\infty} e^{-z^{2} / 2} d z$. Since these formulas are of limited accuracy, we recommend a slightly different form, which will now be justified.

Let

$$
x^{2}=(\operatorname{erf} y)^{2}=\frac{4}{\pi} \int_{0}^{y} e^{-s^{2}} d s \int_{0}^{y} e^{-t^{2}} d t=\frac{4}{\pi} \int_{0}^{y} \int_{0}^{y} e^{-\left(s^{2}+t^{2}\right)} d s d t .
$$

The square over which the integration is performed can be decomposed into two regions, $\psi_{1}$ and $\psi_{2}$, where $\psi_{1}$ is the quarter circle $s^{2}+t^{2} \leqq y^{2}$, and $\psi_{2}$ is the remainder of the square. Converting to polar coordinates, we see that

$$
\frac{4}{\pi} \int_{\psi_{1}} e^{-\left(s^{2}+t^{2}\right)} d s d t=\frac{4}{\pi} \int_{0}^{\pi / 2} \int_{0}^{y} e^{-r^{2}} r d r d \theta=\int_{0}^{y} e^{-r^{2}} 2 r d r=1-e^{-y^{2}} .
$$

Since

$$
\frac{4}{\pi} \int_{\psi_{2}} e^{-\left(s^{2}+t^{2}\right)} d s d t<\frac{4}{\pi} \int_{0}^{\pi / 2} \int_{y}^{y \sqrt{ } 2} e^{-r^{2}} r d r d \theta=e^{-y^{2}}-e^{-2 y^{2}},
$$

this quantity can be neglected relative to $1-e^{-y^{2}}$. Thus $x^{2} \approx 1-e^{-y^{2}}$ and we take $y \approx\left[-\ln \left(1-x^{2}\right)\right]^{1 / 2}$ or

$$
\text { inverf }(x) \approx(-\ln [(1-x)(1+x)])^{1 / 2}
$$

assuming positive $x$. Because of Eqs. (3) and (4) it is possible to preserve accuracy in $1-x$.

To simplify notation, $\beta(x)$ will denote $\left[-\ln \left(1-x^{2}\right)\right]^{1 / 2}$ throughout the remainder of this discussion.

Formula (13) can be improved if we define a new function $R(x)$ such that

$$
\text { inverf }(x)=\beta(x) \cdot R(x) \text {. }
$$

For small $x, \beta(x)$ can be expanded in a power series. Because of this, a power series expansion was also generated for $R(x)$ making use of Eq. (8). The resulting series for $R(x)$ was found to be more strongly convergent than the series (8). Unfortunately, more effort is required to evaluate $\beta(x)$ than to compute the extra terms in (8).

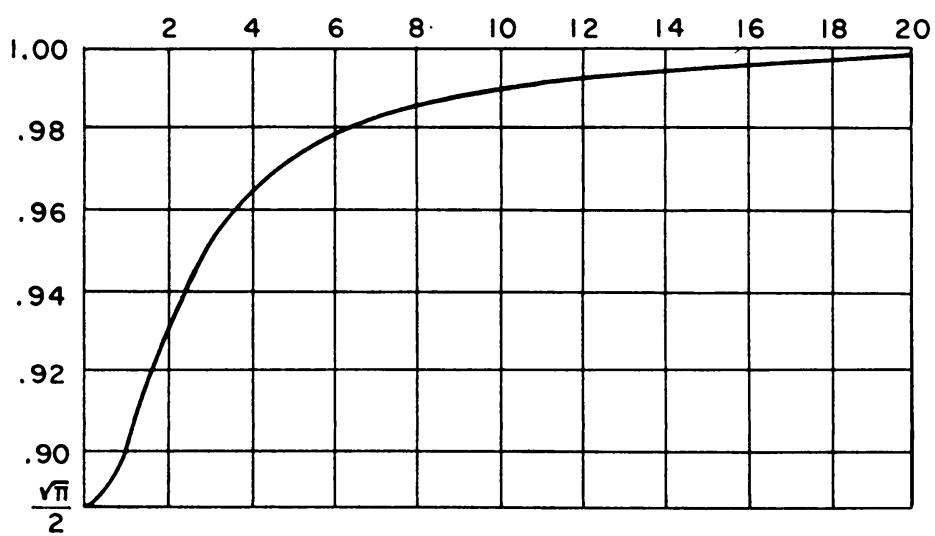

Figure 1. $R(X)$ VS. INVERF $(X)$ 
In Fig. 1 is a plot of $R(x)$ versus $y$. As the graph illustrates, $R(x)$ increases monotonically from $\sqrt{ } \pi / 2$ to 1 as $y$ increases from 0 to $\infty$, showing that the relative error due to formula (13) is never larger than $2 / \sqrt{ } \pi-1$.

The formulas for $R(x)$ which are given below were obtained by applying Chebyshev interpolation [12] to inverf $(x) / \beta(x)$.

For $.8 \leqq x \leqq .9975$,

$$
R(x) \approx \sum_{n=0}^{26} \lambda_{n} T_{n}\left(D_{1} \beta(x)+D_{2}\right),
$$

where

$$
\begin{array}{llllll}
D_{1}=-1.54881 & 30423 & 73261 & 65951 & 2742, \\
D_{2}= & 2.56549 & 01231 & 47816 & 15192 & 8163,
\end{array}
$$

and the coefficients $\lambda_{n}$ are given in Table 4 .

For $25 \cdot 10^{-4} \geqq 1-x \geqq 5 \cdot 10^{-16}$,

$$
R(x) \approx \sum_{n=0}^{37} \delta_{n} T_{n}\left(D_{3} \beta(x)+D_{4}\right)
$$

where

$$
\begin{array}{lllll}
D_{3}=-.55945 & 76313 & 29832 & 32254 & 36913, \\
D_{4}=2.28791 & 57162 & 63357 & 63896 & 5891,
\end{array}
$$

and the coefficients $\delta_{n}$ are given in Table 5 .

For $5 \cdot 10^{-16} \geqq 1-x \geqq 10^{-300}$,

$$
R(x) \approx \sum_{n=0}^{25} \mu_{n} T_{n}\left(D_{5} /(\beta(x))^{1 / 2}+D_{6}\right),
$$

where

$$
\begin{aligned}
& D_{5}=-9.19999 \quad 23588 \quad 30151 \quad 03127 \quad 8420 \text { ， }
\end{aligned}
$$

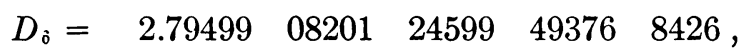

and the coefficients $\mu_{n}$ are given in Table 6 .

Considering the limitations of our formulas, function subroutines, and roundoff errors, these results are not as accurate as the length of the numbers given in Tables 4,5 , and 6 would seem to imply. Twenty-five decimals are given because it is not known which digits are significant.

Test cases which obtained $\epsilon_{2}$ in (11) from equations (14) through (17) showed that $\epsilon_{2}<10^{-22}$.

A more severe test case using equations (3), (4), (14), (15), (16), and (17) which obtained

$$
\epsilon_{3}=\mid \lambda^{-1}[1-\operatorname{erf}(\text { inverf }(1-\lambda))]-1 \mid
$$

showed a larger error, with $\epsilon_{3}<10^{-19}$. 
A. J. STRECOK

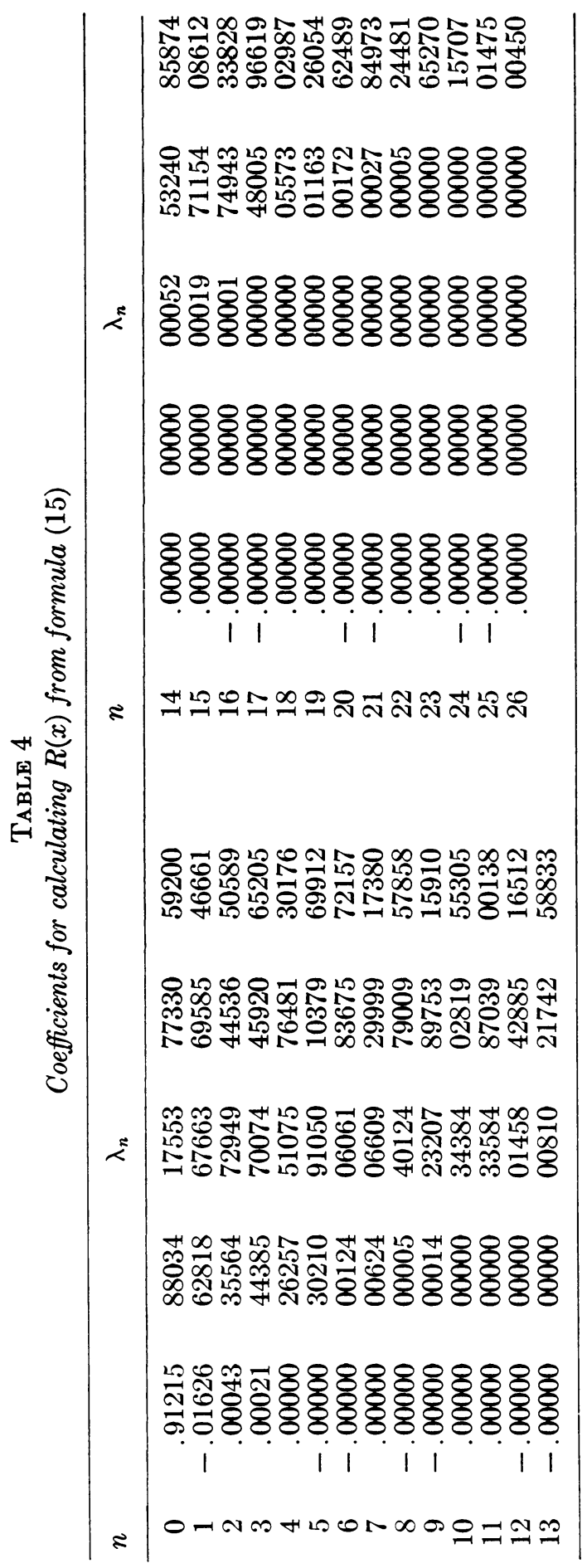




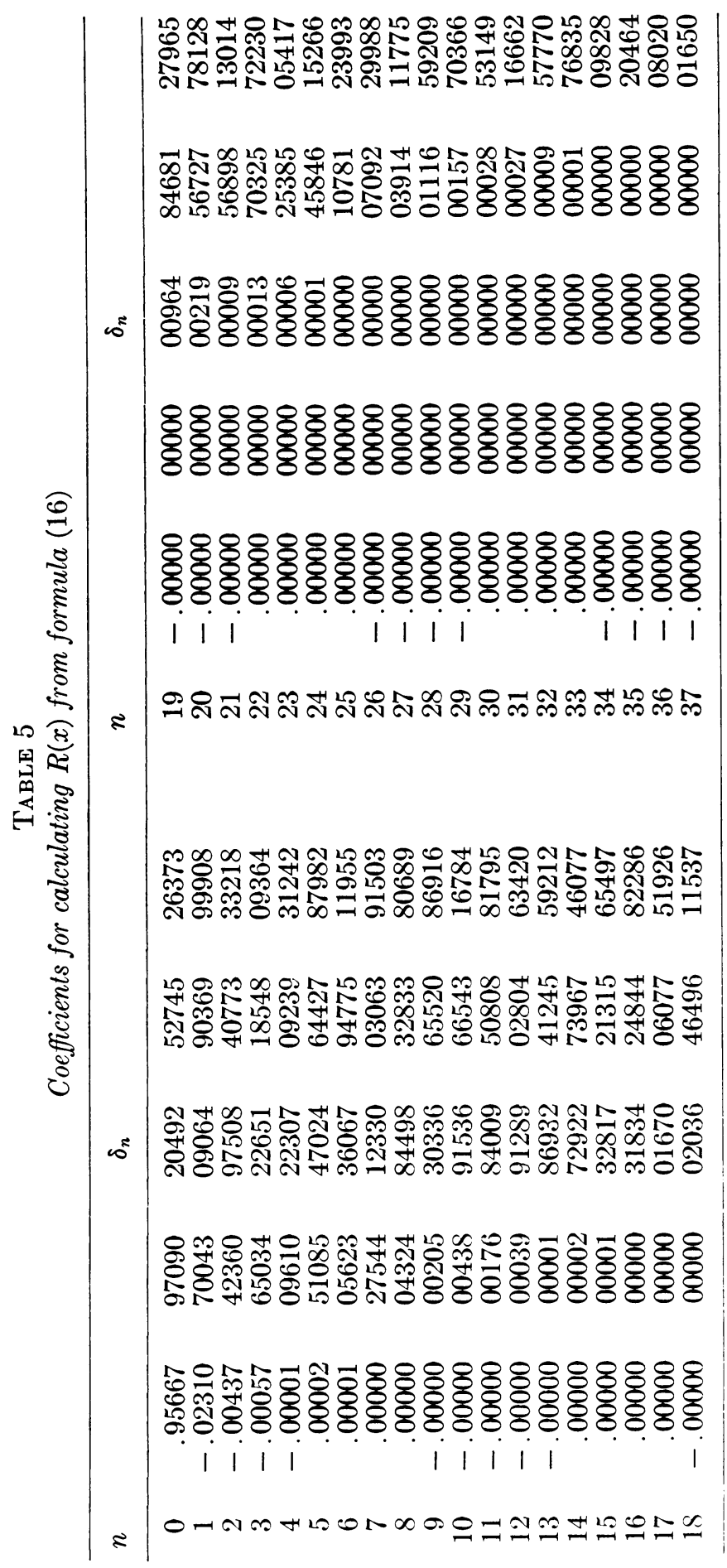




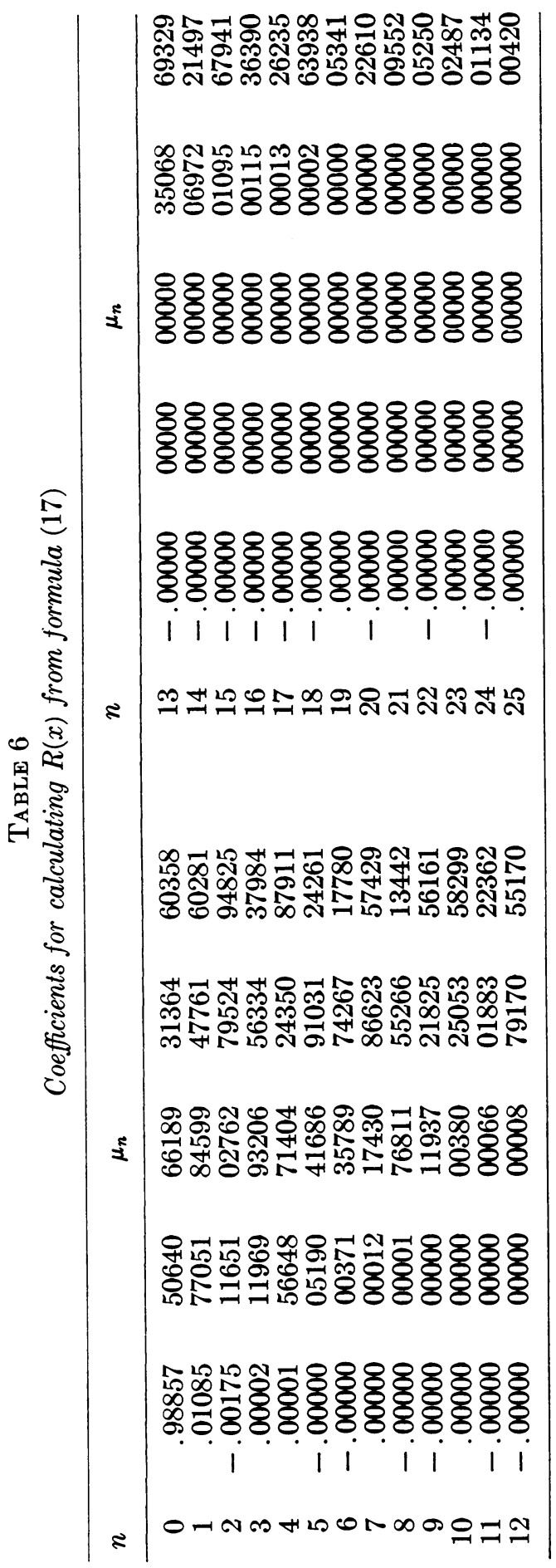


5. Comments on Errors. Since the result produced by the formulas of the preceding section includes an error, Dr. D. Woodward of Argonne contributed some of the ideas discussed below.

Let $y^{*}=y+\epsilon$ assuming $x=$ erf $(y)$ is exact. From Taylor's series and Eqs. (5) and (6) we obtain

$$
\operatorname{inverf}\left(\operatorname{erf}\left(y^{*}\right)\right)=\operatorname{inverf}(\operatorname{erf}(y))+\sqrt{ } \pi e^{y^{2}} h_{1} / 2+\pi\left(y+\theta_{1} \epsilon\right) / 4\left(e^{2\left(y+\theta_{1} \epsilon\right)^{2}} h_{1}^{2}\right)
$$

and

$$
\begin{gathered}
\text { inverfc }\left(\operatorname{erfc}\left(y^{*}\right)\right) \\
=\operatorname{inverfc}(\operatorname{erfc}(y))+\sqrt{ } \pi e^{y^{2}} h_{2} / 2+\pi\left(y+\theta_{2} \epsilon\right) / 4\left(e^{2\left(y+\theta_{2} \epsilon\right)^{2}} h_{2}{ }^{2}\right)
\end{gathered}
$$

where

$$
h_{1}=\operatorname{erf}\left(y^{*}\right)-\operatorname{erf}(y), \quad 0<\theta_{1}<1,
$$

and

$$
h_{2}=\operatorname{erfc}\left(y^{*}\right)-\operatorname{erfc}(y), \quad 0<\theta_{2}<1 .
$$

If $\eta_{m}=\sqrt{ } \pi e^{y^{2}} h_{m} / 2,(m=1,2)$, then Eqs. (18) and (19) can be written as

$$
\epsilon=y^{*}-y=\eta_{m}+\left(y+\theta_{m} \epsilon\right) \exp \left\{2 \theta_{m} \epsilon\left(2 y+\theta_{m} \epsilon\right)\right\} \eta_{m}{ }^{2} .
$$

Equation (20) shows that the error in $y$ is approximately equal in magnitude to $\eta_{m}$ when $\eta_{m}$ is sufficiently small. For $y \leqq 2$, the computer program interpolated for $y^{*}$ subject to the condition that $\left|h_{1}\right|=\left|\operatorname{erf}\left(y^{*}\right)-x\right|<x \cdot 10^{-23}$. Thus $\left|\eta_{1}\right|<\sqrt{ } \pi e^{y^{2}} / 2 \cdot x \cdot 10^{-23}<10^{-21}$. This shows that it is possible to obtain $y$ from $x$ to at least 21 decimal places on the 3600 computer whenever $x \leqq \operatorname{erf}(2)$ is known to at least 24 significant decimal places.

For $y>2, y^{*}$ was obtained with the restriction that

$$
\left|h_{2}\right|=\left|\operatorname{erfc}\left(y^{*}\right)-1+x\right|<(1-x) 10^{-22} .
$$

Then

$$
\left|\eta_{2}\right|<\frac{\sqrt{ } \pi}{2} e^{y^{2}}\left(\frac{2}{\sqrt{ } \pi} \int_{y}^{\infty} e^{-t^{2}} d t 10^{-22}\right)<10^{-22} .
$$

Since $y$ is never larger than 27 for the range under consideration, formula (20) implies that we can obtain $y$ to at least 21 decimal places for $y>2$ whenever erfc $(y)$ is known to at least 22 significant figures.

Since $y^{*}$ is assumed to be larger than .5 , the relative error in $y$ cannot be larger than $2 \epsilon$.

6. Conclusion. Extensive testing with thousands of arguments of 24-decimal significance in the range $0<|x| \leqq 1-10^{-300}$ and $0<|y| \leqq 26.2$ showed that we should expect at least 18-decimal significance in the results of all formulas which were developed in this report.

7. Acknowledgments. In addition to those mentioned previously, the author would like to express deep gratitude to Drs. A. Jaffey, R. F. King, and H. C. 
Thacher, Jr., of Argonne for many valuable suggestions which were incorporated into this report.

Argonne National Laboratory

Argonne, Illinois

1. R. A. Fisher \& E. A. Cornish, "The percentile points of distributions having known cumulants," Technometrics, v. 2, 1960, pp. 209-223.

2. H. GoLDBERG \& H. Levine, "Approximate formulas for the percentage points and normalization of $t$ and $\chi^{2}$," Ann. Math. Statistics, v. 17, 1946, pp. 216-225. MR 8, 42.

3 . J. WISHART, " $\chi^{2}$ probabilities for large numbers of degrees of freedom," Biometrika, v. 43, 1956, pp. 92-95. MR 18, 78.

4. E. PAULSON, "An approximate normalization of the analysis of variance distribution," Ann. Math. Statistics, v. 13, 1942, pp. 233-235. MR 4, 23. \#9626.

5. J. R. PhILIP, "The function inverfe $\theta$," Austral. J. Phys., v. 13, 1960, pp. 13-20. MR 22

6. L. Carlitz, "The inverse of the error function," Pacific J. Math., v. 13, 1963, pp. 459-470. MR $27 \# 3839$.

7. H. KunI, Mathematical Functions, a Description of the Center's 7094 FORTRAN II Mathematical Function Library, University of Chicago Computation Center, February 1966, pp. 205-214.

8. J. B. Rosser, Theory and Application of $\int_{0}^{z} e^{-x^{2}} d x$ and $\int_{0}^{z} e^{-p^{2} y^{2}} d y \int_{0}^{y} e^{-x^{2}} d x$, Part 1: Methods of Computation, ORSD 5861, Mapleton House, Brooklyn, N. Y., 1948, p. 32. MR 10, 267.

9. H. WALL, Analytic Theory of Continued Fractions, Van Nostrand, Princeton, N. J., 1948, p. 358. MR 10, 32 .

10. H. C. THACHER, JR., "Conversion of a power to a series of Chebyshev polynomials," Comm. $A C M$, v. 7,1964 , pp. $181,182$.

11. C. Hastings, Approximations for Digital Computers, Princeton Univ. Press, Princeton, N. J., 1955, pp. 191-192. MR 16, 963.

12. F. B. Hildebrand, Introduction to Numerical Analysis, McGraw-Hill, New York, 1956, pp. 389-395. MR 17, 788. 